\title{
Case Study: Feasibility Analysis of Close-in Proximity Operations Using Angles-only Navigation*
}

\author{
Feng LI, ${ }^{1)}$ Xibin CAO, ${ }^{1 \dagger}{ }^{\dagger}$ Yue You, ${ }^{2)}$ and Jinxiu ZHANG ${ }^{1)}$ \\ ${ }^{1)}$ Research Center of Satellite Technology, Harbin Institute of Technology, Harbin 150001, China \\ ${ }^{2)}$ Naval Research Academy, Beijing 100161, China
}

\begin{abstract}
The feasibility of angles-only navigation (AON)-based close-in proximity operation is studied for application to upcoming small satellite-based uncooperative rendezvous missions. For the relative motion equations described by Yamanaka-Ankersen, the Square-Root Unscented Kalman Filter (SRUKF) is adapted for relative position estimations, considering low-volume/mass, lower-power, simple optical/infrared instruments together with Lidar measurements. Considering simplicity and achievements in engineering, the multi-pulse glideslope guidance law is utilized. Using a linear covariance technique, a complete set of analytical functions are deduced for closed-loop true dispersions and estimated dispersion analysis. Monte Carlo simulation proves the offset observation model proposed provides a good solution for the range-observability dilemma: the range estimation error decreases from an initial decameter-level to a final decimeter-level. Two proximity operation mission trajectories are designed: direct v-bar quasi-linear glide approach for robotic arm capture or net capture, and glide approach (i.e., circumnavigate-glide approach for capture with attitude requirement). Using a well-designed relative approaching guidance profile, the camera operational range can be extended. This enables the operation time of the Lidar to be shortened, or even replaced by an optimal/infrared camera. This is very helpful in saving mass and power for the chaser. The variable-structure SRUKF proposed leads to a more robust trajectory: true dispersion is improved by two orders in the $100 \mathrm{~m}$ range, and more accurate covariance prediction (i.e., all sampled trajectories are inside the ellipse) when compared to the standard SRUKF. The analysis method proposed, which could raise analytical closed-loop linear covariance, is applicable for onboard maneuver planning and real-time closed-loop control error estimation.
\end{abstract}

Key Words: Angles-Only Navigation, Close-in Proximity Operations, Square Root Unscented Kalman Filter (SRUKF)

\section{Introduction}

It is well known that unused spacecraft and debris cause a threat to all operating spacecraft; especially noted is the collision of Iridium 33 with Kosmos-2251 in 2009, which led to the destruction of an active satellite worth tens of millions of dollars. ${ }^{1)}$ The joint advice given by international research institutes and associations is that at least five currently existing large space debris should be removed per year in order to prevent conditions from worsening. For example, considering the condition that EnviSat has a high risk of catastrophic collision, removal capabilities have been developed by the ESA's CleanSpace program. ${ }^{2,3)}$ The same technologies can be applied to other uncooperative rendezvous missions, and can be further extended to autonomous orbital inspection and repair drones in close distance.

With respect to the use of low-cost, simple nano-satellites for active debris removal (ADR), angles-only navigation (AON)-based single-optical/infrared instruments seem to be one practical solution. It is worth mentioning that using $\mathrm{AON}$ in autonomous vehicles is currently a hot research subject. $^{4-14)}$ The growing interest in vision-based autonomous rendezvous and docking has not only resulted in the proposal of experimental satellite missions, but has also resulted in the

(C) 2020 The Japan Society for Aeronautical and Space Sciences

*Received 6 September 2018; final revision received 18 March 2019; accepted for publication 13 June 2019.

†Corresponding author, xbcao@hit.edu.cn development of close-rendezvous technology for small satellites. PRISMA ${ }^{15)}$ is the in-orbit test bed for AON technology. OHB Sweden, ${ }^{16)} \mathrm{CNES}^{17)}$ and $\mathrm{DLR}^{18)}$ have conducted uncooperative rendezvous utilizing AON separately; however, the use of global positioning satellite (GPS) and radio frequency (RF) technologies is still uncertain.

A key challenge for AON is the inherent difficulty to accurately determine the range to and position of an uncooperative target. ${ }^{7)}$ In rigorous terms, the navigation problem can be regarded as an observable problem if it is possible to conduct orbit control maneuvers. Once having the capability of orbit maneuvers, it will be possible to change the relative orbit geometry from the observer to the target. ${ }^{11)}$ Woffinden and Geller ${ }^{6,7)}$ and Grzymisch and Walter ${ }^{14)}$ separately derived some different observability criterion for AON. Spurmann ${ }^{19)}$ proposed the spiraling maneuver method to cover the transition gap from absolute to determine the relative position. Based on minimizing fuel consumption, $\mathrm{Pi}$ and $\mathrm{Bang}^{20)}$ gave the observability constraint at each rendezvous maneuver optimization node and ensured sufficient relative observability during the entire rendezvous maneuver process. All of the past research has considered the observability index as an optimization cost function or constraint, which is only suited for offline and open loop ${ }^{21)}$ navigation processes. However, uncooperative rendezvous is a long-term autonomous mission with modeling and control errors, as adjacent maneuvering interval constraints and data gaps induced by solar eclipses must also be considered. ${ }^{22}$ With the exception of 
observability, there should be a trade-off between safety related to trajectory dispersion and fuel constraints considering satellites that usually have tight mass budget. Considering navigation and control error, Tang et al. ${ }^{23}$ ) and Luo et al. $^{24-26)}$ both proposed an open-loop optimal robust rendezvous planning approach, where a trade-off between fuel expenditure, maneuver time and trajectory robustness was made. To manage the problem of position robustness, velocity robustness and fuel consumption, Li et al. ${ }^{27)}$ presented the closed-loop multiple-objective optimization problem (MOOP). The previous maneuver planning approaches all utilized physical planning and a non-dominated sorting genetic algorithm (NSGA-II). This causes a heavy computational burden, which cannot be used onboard due to limit computational resources.

In this paper, the relative rendezvous trajectory is optimized for AON between the chaser and target satellite. The goal of this paper is to design an online rendezvous maneuver planning method that provides better observability and robustness, and improved fuel efficiency. There is a tradeoff between the relative position robustness, relative velocity robustness and fuel consumption.

The remainder of the paper is organized as follows: "Problem Formulation" addresses the basics of relative dynamics, the traditional and novel camera observation models, Lidar observation model and measurements filtering scheme. "Closed-Loop Linear Covariance Analysis" introduces the analytical linear covariance method to quantify the navigated dispersion and true dispersions for closed-loop relative tra- jectory. In "Numerical Simulation," three simulation cases are given: Case 1 is designed to validate and compare the effectiveness of close-range AON observation model; Case 2 is used to validate the feasibility of AON, a v-bar quasi-linear glide approaching scenario is built, and two different range measurement accuracy Lidar-based navigation methods are selected for comparison; and Case 3 is utilized to compare the influence of nominal trajectory on control accuracy, trajectory robustness and fuel consumption using an approachcircumnavigate-approach trajectory. The "Conclusion" presents concluding remarks for this study.

\section{Problem Formulation}

\subsection{Dynamic modeling}

The origin of a rotating local vertical, local horizontal $(\mathrm{LVLH})$ reference frame $\left(O_{t}-x y z\right)$ is collocated with the target. The relative position and velocity of the chaser with respect to the target in the $\left(O_{t}-x y z\right)$ coordinate are defined by $\boldsymbol{r}$ and $\boldsymbol{v}$. The relative motion equation with respect to elliptical orbit is the Tschauner-Hemper (TH) equation, ${ }^{28)}$ whose homogeneous solution is called the Yamanaka-Ankersen state transition matrix, ${ }^{29)}$

$\boldsymbol{\Phi}\left(t, t_{0}\right)=\left[\begin{array}{c:c}\boldsymbol{\Phi}_{r r}\left(t, t_{0}\right) & \boldsymbol{\Phi}_{r v}\left(t, t_{0}\right) \\ \hdashline \boldsymbol{\Phi}_{v r}\left(t, t_{0}\right) & \boldsymbol{\Phi}_{v v}\left(t, t_{0}\right)\end{array}\right]=\boldsymbol{\Phi}_{\theta}(f) \boldsymbol{\Phi}_{\theta}^{-1}\left(f_{0}\right)$

where, $f_{0}$ and $f$ are the target's true anomalies at $t_{0}$ and $t$. The respective expressions of $\boldsymbol{\Phi}_{\theta}^{-1}\left(f_{0}\right)$ and $\boldsymbol{\Phi}_{\theta}(f)$ are as follows:

$$
\begin{aligned}
& \boldsymbol{\Phi}_{\theta}^{-1}\left(f_{0}\right)=\frac{1}{\lambda^{2}} \times\left[\begin{array}{ccc:ccc}
-3 s \frac{k+e^{2}}{k^{2}} & c-2 e & 0 & -s \frac{k+1}{k} & 0 & 0 \\
-3\left(e+\frac{c}{k}\right) & -s & 0 & -\left(c \frac{k+1}{k}+e\right) & 0 & 0 \\
\hdashline 3 k-\lambda^{2} & e s & 0 & k^{2} & 0 & 0 \\
-3 e s \frac{k+1}{k^{2}} & -2+e c & \lambda^{2} & -e s \frac{k+1}{k} & 0 & 0 \\
0 & 0 & 0 & 0 & \lambda^{2} c & -\lambda^{2} s \\
0 & 0 & 0 & 0 & \lambda^{2} s & \lambda^{2} c
\end{array}\right] \text {, } \\
& \boldsymbol{\Phi}_{\theta}(f)=\left[\begin{array}{ccc:ccc}
s & c & 2-3 e s I & 0 & 0 & 0 \\
s^{\prime} & c^{\prime} & -3 e\left(s^{\prime} I+\frac{s}{k^{2}}\right) & 0 & 0 & 0 \\
c\left(1+\frac{1}{k}\right) & -s\left(1+\frac{1}{k}\right) & -3 k^{2} I & 1 & 0 & 0 \\
\hdashline-2 s & e-2 c & -3(1-2 e s I) & 0 & 0 & 0 \\
0 & 0 & 0 & 0 & c & s \\
0 & 0 & 0 & 0 & -s & c
\end{array}\right]
\end{aligned}
$$

with

$$
k=1+e \cos f, c=k \cos f, s=k \sin f, I=\int_{f_{0}}^{f} \frac{1}{k^{2}} \mathrm{~d} u=\frac{\mu^{2}}{h^{3}}\left(t-t_{0}\right) \text { and } \lambda=\sqrt{1-e^{2}} .
$$


Furthermore, $e$ is the eccentricity of target orbit, $\mu$ is the Earth's gravitational constant, and $h$ is the norm of angular momentum. $c^{\prime}$ and $s^{\prime}$ indicate the first derivatives with respect to $f$.

These solutions are rewritten in discrete form for impulsive input $\Delta \boldsymbol{v}_{k}$ as

$$
\boldsymbol{x}_{t}=\boldsymbol{\Phi}\left(t, t_{0}\right) \boldsymbol{x}_{0}+\delta\left(t_{k}\right) \Delta \boldsymbol{v}_{k}
$$

where, $\boldsymbol{x}_{0}=\left[\boldsymbol{r}_{0}, \boldsymbol{v}_{0}\right]^{\mathrm{T}}$ and $\boldsymbol{x}_{t}=\left[\boldsymbol{r}_{t}, \boldsymbol{v}_{t}\right]^{\mathrm{T}}$ are the relative position and velocity of the chaser at initial time $t_{0}$ and any time $t$, respectively. $\delta\left(t_{k}\right)$ is the Dirac function.

\subsection{Camera observation modeling}

Assuming that camera measurement frame $O_{m}-x_{m} y_{m} z_{m}$ is aligned with the camera's focal plane, its orientation is known and constant. The pixel location of the target is utilized to form a line of sight (LOS) vector to the camera, which is depicted in the $O_{m}-x_{m} y_{m} z_{m}$ frame and defined as $\boldsymbol{l}=\left[\begin{array}{lll}x_{m} & y_{m} & z_{m}\end{array}\right]^{\mathrm{T}}$. The state transformation, from LVLH to the $O_{m}-x_{m} y_{m} z_{m}$ frame, can be given using previous information of inertial attitude, position and velocity. The LOS measurement expressed in the LVLH frame can be utilized to formulate the angle/bearing-only measurement equation, which is rewritten as:

$\boldsymbol{Z}=\left[\begin{array}{c}\varepsilon \\ \theta\end{array}\right]=\boldsymbol{h}(\boldsymbol{x})=\left[\begin{array}{c}\arctan \left(\frac{x \sqrt{x^{2}+y^{2}}+z\left(\rho_{t}-z\right)}{x\left(\rho_{t}-z\right)-z \sqrt{x^{2}+y^{2}}}\right) \\ \arctan \left(\frac{-y}{\sqrt{x^{2}+z^{2}}}\right)\end{array}\right]$

where, $\varepsilon$ and $\theta$ are the measurement values of elevation and azimuth angles, respectively, and $\rho_{t}$ is the average radius of the target orbit.

The measurement-sensitive matrix $\boldsymbol{H}$ can be further obtained by differentiating Eq. (3) with respect to state $\boldsymbol{x}$ using

$$
\boldsymbol{H}=\frac{\partial \boldsymbol{h}(\boldsymbol{x})}{\partial \boldsymbol{x}^{\mathrm{T}}}=\left[\begin{array}{lll:l}
h_{11} & h_{12} & h_{13} & \mathbf{0}_{2 \times 3} \\
h_{21} & h_{22} & h_{23} &
\end{array}\right]
$$

where, the respective expressions of $\boldsymbol{H}$ are as follows:

$$
\left\{\begin{array}{l}
h_{11}=\frac{x\left(\rho_{t}-z\right)}{\sqrt{x^{2}+y^{2}}\left[x^{2}+y^{2}+\left(\rho_{t}-z\right)^{2}\right]}-\frac{z}{x^{2}+z^{2}} \\
h_{21}=\frac{x y}{\sqrt{x^{2}+z^{2}}\left(x^{2}+y^{2}+z^{2}\right)} \\
h_{12}=\frac{y\left(\rho_{t}-z\right)}{\sqrt{x^{2}+y^{2}}\left[x^{2}+y^{2}+\left(\rho_{t}-z\right)^{2}\right]} \\
h_{22}=\frac{-\left(x^{2}+z^{2}\right)}{\sqrt{x^{2}+z^{2}}\left(x^{2}+y^{2}+z^{2}\right)} \\
h_{13}=\frac{x^{2}+y^{2}}{\sqrt{x^{2}+y^{2}}\left[x^{2}+y^{2}+\left(\rho_{t}-z\right)^{2}\right]}-\frac{x}{x^{2}+z^{2}} \\
h_{23}=\frac{y z}{\sqrt{x^{2}+z^{2}}\left(x^{2}+y^{2}+z^{2}\right)}
\end{array}\right.
$$

When camera offset is neglected, there exists a widely acknowledged range observability problem. This is when the chaser maintains v-bar relative station-keeping, as the relative range cannot be estimated at all. But v-bar relative position-keeping is very useful in space rendezvous for on-orbit servicing, close operation, formation flying and silent investigation. It can be used for parking, task process coordinating, emergency handling, maneuver preparation and longterm tracking. In the past, decoupled out-of-plane oscillation was used to increase observability. On one hand, the generation and elimination of out-of-plane oscillations require the use of a paired normal maneuver, which brings additional fuel consumption. On the other hand, due to the limitation in navigation precision, realizing the precise control of the normal oscillation amplitude motion is very difficult. Here, a novel AON method for close-range ( $\rho<100 \mathrm{~m}$ ) observation is developed. A decimeter offset is deliberately added for camera installation assuming projection in the LVLH frame is $\boldsymbol{r}_{c m}=\left[\begin{array}{llll}\delta x_{c m} & \delta y_{c m} & \delta z_{c m}\end{array}\right]^{\mathrm{T}}$ and then $\left\|\boldsymbol{r}_{c m}\right\| / \rho \geq$ $10^{-3}$. When the chaser is at the $\mathrm{v}$-bar relative station-keeping point, its relative state is $x^{2}=\rho, y=z=0$. Considering the camera offset $\boldsymbol{r}_{c m}$, the new measurement angles are calculated by substituting $x, y$ and $z$ in Eq. (3) with $\tilde{x}=x+\delta x_{c m}$, $\tilde{y}=\delta y_{c m}$ and $\tilde{z}=\delta z_{c m}$, and noticing $\tilde{x}^{2}+\tilde{y}^{2} \approx \tilde{x}^{2}+\tilde{z}^{2} \approx$ $\tilde{x}^{2}+\tilde{y}^{2}+\tilde{z}^{2}=\rho^{2}, \rho_{t} \gg \rho$. Then, the close-range observation model is as follows:

$$
\boldsymbol{Z}_{\text {close }}=\left[\begin{array}{c}
\varepsilon \\
\theta
\end{array}\right]=\boldsymbol{h}(\boldsymbol{x})=\left[\begin{array}{c}
\arctan \left(\frac{\tilde{z}}{\tilde{x}}\right) \\
\arctan \left(\frac{-\tilde{y}}{\sqrt{\tilde{x}^{2}+\tilde{z}^{2}}}\right)
\end{array}\right]
$$

The corresponding observation sensitivity matrix is

$$
\boldsymbol{H}=\left[\begin{array}{rrr:r}
0 & 0 & \frac{1}{\rho} & \\
0 & -\frac{1}{\rho} & 0 & \\
& & &
\end{array}\right]
$$

From Eq. (7), we know the offset projection in the $x$ direction has no effect on improving angular measurement, but offset projection in the $y$ and $z$ directions has an equivalent effect. This conclusion is based on the assumption that $\boldsymbol{r}_{c m}$ maintains a fixed attitude relative to the LVLH frame all of the time. In this way, the well-documented unobservable v-bar relative station-keeping problem becomes observable without external fuel consumption.

\subsection{Lidar observation modeling}

The Lidar measurement frame $\left(O_{L m}-x_{L m} y_{L m} z_{L m}\right)$ is assumed to coincide with the camera measurement frame $\left(O_{m}-x_{m} y_{m} z_{m}\right)$. Accordingly, the relative observation geometry is same as shown in Fig. 1. The Lidar measurement equation, which provides the measurement angles and range to target, is expressed in the LVLH frame as follows: 


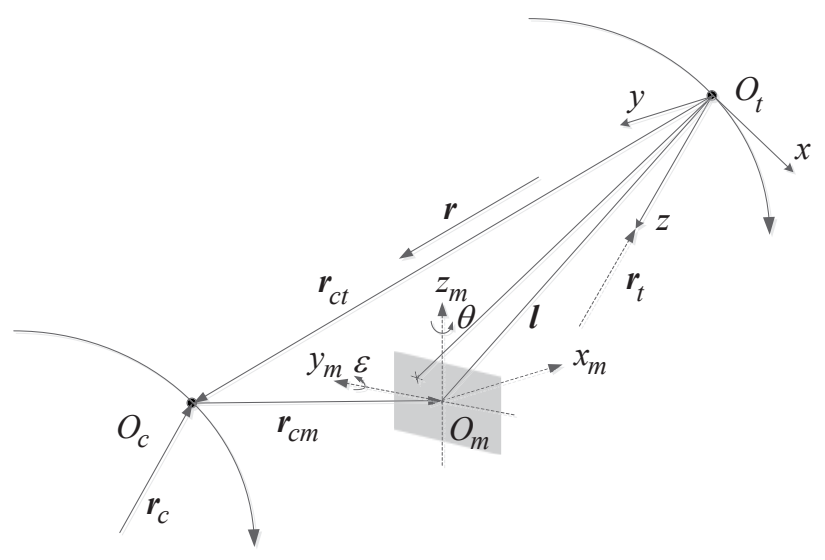

Fig. 1. Relative observation geometry.

$$
\begin{aligned}
\boldsymbol{Z}_{L} & =\left[\begin{array}{l}
\rho \\
\varepsilon \\
\theta
\end{array}\right] \\
& =\boldsymbol{h}_{L}(\boldsymbol{x}) \\
& =\left[\begin{array}{c}
\sqrt{x^{2}+y^{2}+z^{2}} \\
\arctan \left(\frac{x \sqrt{x^{2}+y^{2}}+z\left(\rho_{t}-z\right)}{x\left(\rho_{t}-z\right)-z \sqrt{x^{2}+y^{2}}}\right) \\
\arctan \left(\frac{-y}{\sqrt{x^{2}+z^{2}}}\right)
\end{array}\right]
\end{aligned}
$$

where, $\rho$ is the relative range. The measurement-sensitive matrix $\boldsymbol{H}$ can be further obtained by differentiating Eq. (6) with respect to state $\boldsymbol{x}$ using

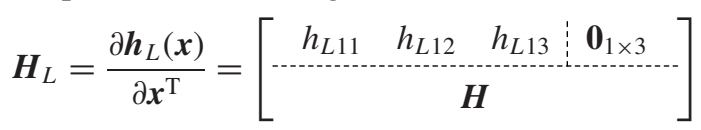

where, the respective expressions of $h_{L 11}, h_{L 12}$ and $h_{L 13}$ are as follows:

$$
\left\{\begin{array}{l}
h_{L 11}=\frac{x}{\sqrt{x^{2}+y^{2}+z^{2}}} \\
h_{L 12}=\frac{y}{\sqrt{x^{2}+y^{2}+z^{2}}} \\
h_{L 13}=\frac{z}{\sqrt{x^{2}+y^{2}+z^{2}}}
\end{array}\right.
$$

\subsection{Measurements filtering scheme}

SRUKF $^{30-35)}$ is applied as the measurement filtering method, utilizing a deterministic "sampling" scheme to calculate mean and covariance terms of the relative state. $2 L+1$ (i.e., $L$ is the state dimension) sigma points are propagated through the true nonlinearity without approximation, and then a weighted mean and covariance are taken. This method results in approximations that are accurate to the third-order (Taylor series expansion) for Gaussian inputs for all nonlinearities. With respect to non-Gaussian inputs, approximations are accurate to at least the second-order. ${ }^{31)}$
In contrast, the linearization approach of the EKF results only in first-order accuracy.

The implementation for $\mathrm{AON}$ is given as follows: Initialization:

$$
\begin{aligned}
& \hat{\boldsymbol{x}}_{0}=\boldsymbol{E}\left[\boldsymbol{x}_{0}\right] \\
& \boldsymbol{S}_{0}=\operatorname{chol}\left\{\boldsymbol{E}\left[\left(\boldsymbol{x}_{0}-\hat{\boldsymbol{x}}_{0}\right)\left(\boldsymbol{x}_{0}-\hat{\boldsymbol{x}}_{0}\right)^{\mathrm{T}}\right]\right\}
\end{aligned}
$$

where, $\operatorname{chol}\{\cdot\}$ stands for the Cholesky decomposition.

For $k \in\{1, \cdots, \infty\}$, calculate the sigma points:

$$
\chi_{k-1}=\left[\begin{array}{lll}
\hat{\boldsymbol{x}}_{k-1} & \hat{\boldsymbol{x}}_{k-1}+\eta \boldsymbol{S}_{k} & \hat{\boldsymbol{x}}_{k-1}-\eta \boldsymbol{S}_{k}
\end{array}\right]
$$

Time update:

$$
\begin{aligned}
& \hat{\boldsymbol{\chi}}_{k}=\boldsymbol{f}\left(\hat{\boldsymbol{\chi}}_{k-1}, \boldsymbol{u}_{k-1}\right)+\boldsymbol{w}_{k-1} \\
& \hat{\boldsymbol{x}}_{k}^{-}=\sum_{i=0}^{2 L} W_{i}^{m} \hat{\boldsymbol{\chi}}_{i, k} \\
& \hat{\boldsymbol{S}}_{k}^{-}=\operatorname{qr}\left\{\left[\sqrt{W_{1}^{c}}\left(\hat{\chi}_{1: 2 L, k}-\hat{\boldsymbol{x}}_{k}^{-}\right) \sqrt{\boldsymbol{Q}_{k-1}}\right]\right\} \\
& \hat{\boldsymbol{S}}_{k}^{-}=\operatorname{cholupdate}\left\{\hat{\boldsymbol{S}}_{k}^{-}, \hat{\chi}_{0, k}-\hat{\boldsymbol{x}}_{k}^{-}, W_{0}^{c}\right\} \\
& \hat{\boldsymbol{Z}}_{k}=\boldsymbol{h}\left(\hat{\boldsymbol{\chi}}_{k}\right)+\boldsymbol{v}_{k-1} \\
& \hat{\boldsymbol{z}}_{k}=\sum_{i=0}^{2 L} W_{i}^{m} \hat{\boldsymbol{Z}}_{i, k}
\end{aligned}
$$

The shorthand notation $\operatorname{qr}\{\cdot\}$ is used to describe the QR decomposition of a matrix, cholupdate $\{\cdot\}$ to donate Cholesky factor updating.

Measurement update:

$$
\begin{aligned}
& \boldsymbol{S}_{\hat{z}_{k}}=\operatorname{qr}\left\{\left[\sqrt{W_{1}^{c}}\left(\hat{\boldsymbol{Z}}_{1: 2 L, k}-\hat{\boldsymbol{z}}_{k}\right)\right] \sqrt{\boldsymbol{R}_{k-1}}\right\} \\
& \boldsymbol{S}_{\hat{z}_{k}}=\text { cholupdate }\left\{\boldsymbol{S}_{\hat{z}_{k}}, \hat{\boldsymbol{Z}}_{0, k}-\hat{\boldsymbol{z}}_{k}, W_{0}^{c}\right\} \\
& \boldsymbol{P}_{\hat{\boldsymbol{x}}_{k} \hat{z}_{k}}=\sum_{i=0}^{2 L} W_{i}^{c}\left[\hat{\boldsymbol{\chi}}_{i, k}-\hat{\boldsymbol{x}}_{k}^{-}\right]\left[\hat{\boldsymbol{Z}}_{i, k}-\hat{z}_{k}\right]^{\mathrm{T}} \\
& \boldsymbol{K}_{k}=\left(\boldsymbol{P}_{\hat{\boldsymbol{x}}_{k} \hat{z}_{k}} / \boldsymbol{S}_{\hat{z}_{k}}^{\mathrm{T}}\right) / \boldsymbol{S}_{\hat{z}_{k}} \\
& \hat{\boldsymbol{x}}_{k}=\hat{\boldsymbol{x}}_{k}^{-}+\boldsymbol{K}_{k}\left(\boldsymbol{z}_{k}-\hat{\boldsymbol{z}}_{k}\right) \\
& \boldsymbol{U}=\boldsymbol{K}_{k} \boldsymbol{S}_{\hat{z}_{k}} \\
& \boldsymbol{S}_{\hat{\boldsymbol{z}}_{k}}=\operatorname{cholupdate}\left\{\boldsymbol{S}_{\hat{z}_{k}}, \boldsymbol{U},-1\right\}
\end{aligned}
$$

where, $\left\{W_{i}\right\}$ is a set of scalar weights, $W_{i}^{m}$ is the mean weight and $W_{i}^{c}$ is the covariance weight calculated as follows:

$$
\left\{\begin{array}{l}
W_{0}^{m}=\lambda /(\lambda+L) \\
W_{0}^{c}=\lambda /(\lambda+L)+\left(1-\alpha^{2}+\beta\right) \\
W_{i}^{m}=W_{i}^{c}=1 /\{2(L+\lambda)\}, \quad i=1, \cdots, 2 L
\end{array}\right.
$$

where,

$$
\lambda=L\left(\alpha^{2}-1\right) \text { and } \eta=\sqrt{(L+\lambda)} .
$$

$\alpha$ determines the spread of the sigma points around $\hat{\boldsymbol{x}}$ and is set to $10^{-4} \leq \alpha \leq 1$. $\beta$ is utilized to incorporate prior knowledge of the distribution of $\boldsymbol{x}$ (i.e., for Gaussian distributions, $\beta=2$ is optimal). Additionally, note that we define the linear algebra operation of adding a column vector to a matrix (i.e., $\boldsymbol{A} \pm \boldsymbol{u}$ as the addition of the vector to each column of the 


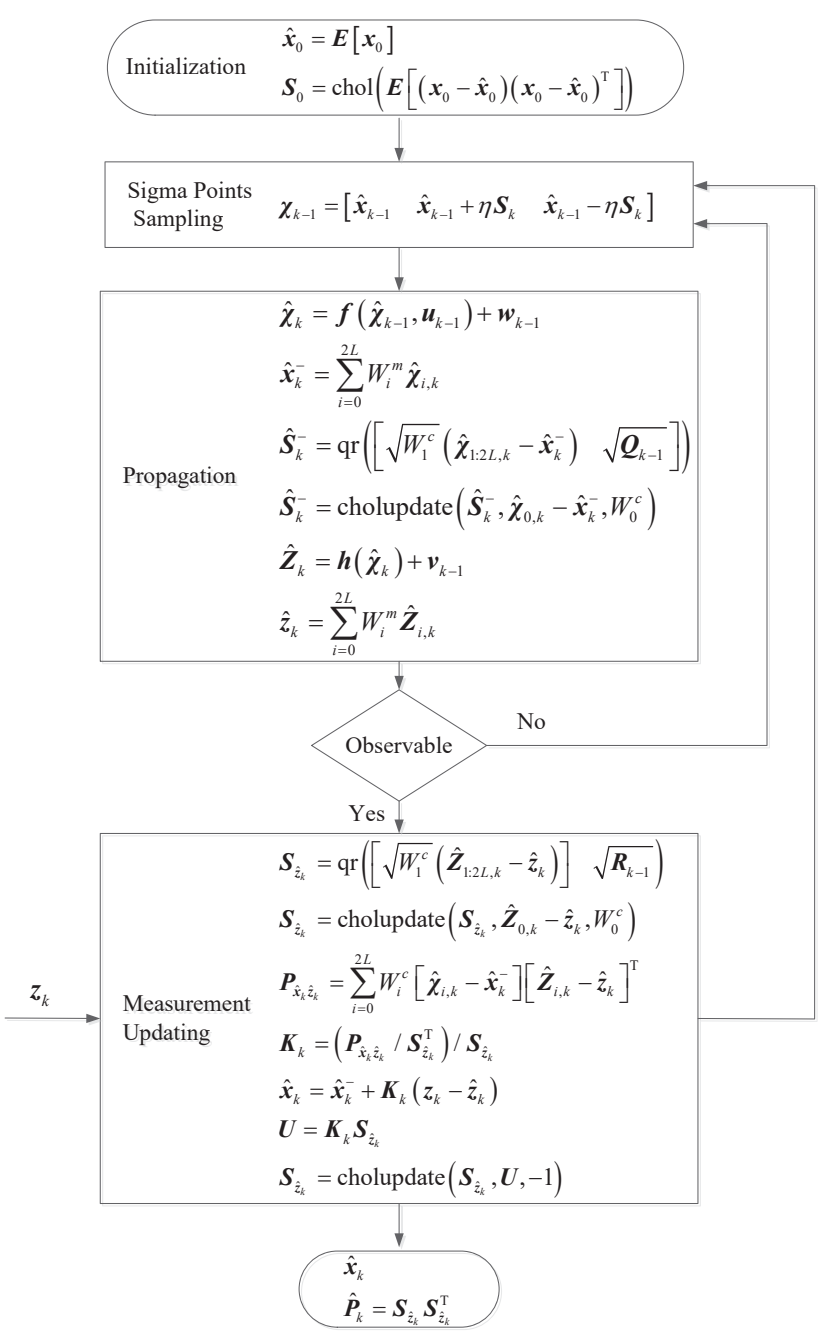

Fig. 2. Filtering equations flow diagram of SRUKF.

matrix). Unlike the EKF, no explicit derivatives need to be calculated.

To improve the robustness and accuracy of the filter, a variable-structure SRUKF is developed in this paper, and the parameters are tuned as follows:

$$
\left\{\begin{array}{l}
\boldsymbol{S}_{E 0}=S_{I E F} \boldsymbol{S}_{0} \\
\boldsymbol{Q}=Q_{I E F} \boldsymbol{Q}_{0}+Q_{E F} \boldsymbol{Q}_{0} \cdot \exp \left(Q_{D F} t\right) \\
\boldsymbol{R}=R_{I E F} \boldsymbol{R}_{0}+R_{E F} \boldsymbol{R}_{0} \cdot \exp \left(R_{D F} t\right)
\end{array}\right.
$$

where, $S_{I E F}, Q_{I E F}$ and $R_{I E F}$ are the initial enlargement factors of priori navigation error covariance Cholesky decomposition factor $S_{0}$, priori process noise covariance $\boldsymbol{Q}_{0}$ and priori measure noise covariance $\boldsymbol{R}_{0}$, respectively. $Q_{E F}$ and $R_{E F}$ are enlargement factors of $\boldsymbol{Q}_{0}$ and $\boldsymbol{R}_{0}$, respectively. $Q_{D F}$ and $R_{D F}$ are the exponential time varying decay factors, maintaining the relationship as follows:

$$
\left\{\begin{array}{l}
R_{E F} \cdot \exp \left(Q_{D F} t_{f}\right)=1 \\
Q_{E F} \cdot \exp \left(R_{D F} t_{f}\right)=1
\end{array}\right.
$$

where, $t_{f}$ is the total filtering time. Figure 2 gives the filtering equations flow diagram of SRUKF.

\section{Closed-Loop Linear Covariance Analysis}

The linear covariance analysis approach ${ }^{36,37)}$ is utilized to derive an analytical closed-loop control error analysis model. Aiming to obtain a clear mathematical formulation, the real, estimated and nominal relative states are noted as $\boldsymbol{x}, \hat{\boldsymbol{x}}$ and $\overline{\boldsymbol{x}}$, respectively. An augmented error state vector $\boldsymbol{X}$ is defined, which contains the true dispersions, $\delta \boldsymbol{x}=\boldsymbol{x}-\overline{\boldsymbol{x}}$, and navigated dispersions, $\delta \hat{\boldsymbol{x}}=\hat{\boldsymbol{x}}-\boldsymbol{x}$, as follows:

$$
\boldsymbol{X}=\left[\begin{array}{l}
\delta \boldsymbol{x} \\
\delta \hat{\boldsymbol{x}}
\end{array}\right]
$$

The covariance of $\boldsymbol{X}$ is $\boldsymbol{\Omega}=E\left[\boldsymbol{X} \boldsymbol{X}^{\mathrm{T}}\right]$, and initialized as

$$
\boldsymbol{\Omega}_{0}=\left[\begin{array}{c:c}
\boldsymbol{P}_{0} & \mathbf{0}_{6 \times 6} \\
\hdashline \mathbf{0}_{6 \times 6} & \hat{\boldsymbol{P}}_{0}
\end{array}\right]
$$

where, $\boldsymbol{P}_{0}$ is the priori covariance matrix of true trajectory dispersion and $\hat{\boldsymbol{P}}_{0}$ is the priori covariance matrix of navigated dispersions.

The complete closed-loop control process of autonomous rendezvous includes the following three sections. They are propagate time, update measurement and correct control, which are detailed in this section.

\subsection{Propagate time}

For $k \in\{1, \cdots, \infty\}$, the augmented state vector and its covariance are propagated as follows:

$$
\begin{aligned}
& \boldsymbol{X}_{k}^{-}=\boldsymbol{\Psi}_{k \mid k-1} \boldsymbol{X}_{k-1}^{+} \\
& \boldsymbol{\Omega}_{k \mid k-1}=\boldsymbol{\Psi}_{k \mid k-1} \boldsymbol{\Omega}_{k-1}^{+c} \boldsymbol{\Psi}_{k \mid k-1}^{\mathrm{T}}+\boldsymbol{W}_{k-1} \boldsymbol{Q}_{k-1} \boldsymbol{W}_{k-1}^{\mathrm{T}}
\end{aligned}
$$

where,

$$
\boldsymbol{W}_{k-1}=\left[\begin{array}{c}
\boldsymbol{I}_{6 \times 6} \\
\mathbf{0}_{6 \times 6}
\end{array}\right],
$$

and $\boldsymbol{\Psi}_{k \mid k-1}$ is the augmented state transfer matrix, which is given as:

$$
\boldsymbol{\Psi}_{k \mid k-1}=\left[\begin{array}{c:c}
\boldsymbol{\Phi}\left(t_{k}, t_{k-1}\right) & \mathbf{0}_{6 \times 6} \\
\hdashline \mathbf{0}_{6 \times 6} & \boldsymbol{\Phi}\left(t_{k}, t_{k-1}\right)
\end{array}\right]
$$

\subsection{Update measurement}

The augmented state vector and covariance are updated as follows:

$$
\begin{aligned}
& \boldsymbol{X}_{k}^{-}=\boldsymbol{X}_{k \mid k-1}^{-}+\boldsymbol{K}_{k}\left(\boldsymbol{Z}_{k}-\boldsymbol{H}_{k} \boldsymbol{X}_{k \mid k-1}^{-}\right) \\
& \boldsymbol{\Omega}_{k}^{-c}=\boldsymbol{C}_{k} \boldsymbol{\Omega}_{k \mid k-1} \boldsymbol{C}_{k}^{\mathrm{T}}+\boldsymbol{E}_{k} \boldsymbol{R}_{k} \boldsymbol{E}_{k}^{\mathrm{T}}
\end{aligned}
$$

where, the Kalman gain, $\boldsymbol{K}_{k}$, is achieved in Eq. (14).

\subsection{Correct control}

Here, the general multi-pulse glideslope guidance $\mathrm{law}^{38)}$ is utilized for close-in proximity operations. Figure 3 shows a target in a circular orbit and the associated target's LVLH frame. When $t=0$, the chaser satellite is located at $\boldsymbol{r}_{0}$, with its relative velocity equal to $\boldsymbol{v}_{0}^{-}$. The chaser is required to arrive at $\boldsymbol{r}_{T}$ in transfer time $T$ at a velocity specified hereafter. A straight line from $\boldsymbol{r}_{0}$ to $\boldsymbol{r}_{T}$, denoted by vector $\boldsymbol{\rho}$ in Fig. 3 , is the natural commanded path. Let $\boldsymbol{r}_{c}(t)$ measured from the target center of mass be the commanded location of the chaser on this path at time $t, 0 \leq t \leq T$. Then, the boundary values 


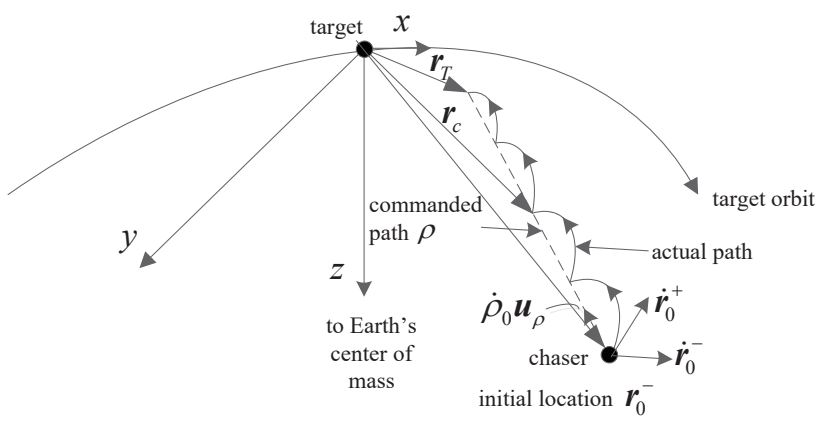

Fig. 3. Three-axis multi-pulse decelerating inbound glideslope.

of $\boldsymbol{r}_{c}(t)$ are $\boldsymbol{r}_{c}(0)=\boldsymbol{r}_{0}$ and $\boldsymbol{r}_{c}(T)=\boldsymbol{r}_{T}$. The vector $\boldsymbol{\rho}(t)$ emanates from the tip of the vector $\boldsymbol{r}_{T}$ and defines the commanded location of the chaser on the straight path from $\boldsymbol{r}_{0}$ to $\boldsymbol{r}_{T}$. The boundary conditions of $\rho(t)$ are $\rho(0)=$ $\boldsymbol{r}_{0}-\boldsymbol{r}_{T} \triangleq \rho_{0}, \rho(T)=0$, and at any time $t, \rho(t)=\boldsymbol{r}_{c}(t)-\boldsymbol{r}_{T}$, where, $\rho(T)$ is defined as $\rho(t)$ at time $T$. Considering $\boldsymbol{r}_{0} \triangleq\left[\begin{array}{lll}x_{0} & y_{0} & z_{0}\end{array}\right]^{\mathrm{T}}$ and $\boldsymbol{r}_{T} \triangleq\left[\begin{array}{lll}x_{T} & y_{T} & z_{T}\end{array}\right]^{\mathrm{T}}$, the direction cosines of the vector $\rho$ are described as $\cos \alpha=\left(x_{0}-x_{T}\right) / \rho_{0}, \cos \beta=\left(y_{0}-y_{T}\right) / \rho_{0}$ and $\cos \gamma=$ $\left(z_{0}-z_{T}\right) / \rho_{0}$, where, $\rho_{0}=\left|\rho_{0}\right|$. The direction of the straight path is given by the unit vector $\boldsymbol{u}_{\rho}$

$$
\boldsymbol{u}_{\rho}=\left[\begin{array}{lll}
\cos \alpha & \cos \beta & \cos \gamma
\end{array}\right]^{\mathrm{T}}
$$

and the scalar distance $\rho$ (i.e., distance to go) along the vector $\rho$ is $\boldsymbol{\rho}=\rho \boldsymbol{u}_{\rho}$. The glideslope guides the distance to go $\rho$ as a function of time $\rho(t)$, so that the chaser is commanded to reach $\boldsymbol{r}_{T}$ from $\boldsymbol{r}_{0}$ in a period $T$ with the arrival commanded velocity $\dot{\rho}_{T} \boldsymbol{u}_{\rho}$, where, $\dot{\rho}_{T}$ less than "0," is predetermined a safe relative speed of the chaser at distance $\left|\boldsymbol{r}_{T}\right|$ from the target.

As the distance to go $\rho$ decreases, the speed $\dot{\rho}$ must decrease. Here, $\dot{\rho}$ is achieved by differentiating $\rho$, treating the LVLH frame as an inertial nonrotating frame. The following linear relationship between $\rho$ and $\dot{\rho}$ is assumed:

$$
\dot{\rho}=a \rho+\dot{\rho}_{T}
$$

where, the parameter $a$ (per second), yet to be determined, is the slope of $\dot{\rho}$ vs $\rho$. The boundary conditions of $\rho$ and $\dot{\rho}$ are at $t=0$,

$$
\rho=\rho_{0}, \quad \dot{\rho}=\dot{\rho}_{0}<0
$$

and at $t=T$,

$$
\rho=0, \quad \dot{\rho}=\dot{\rho}_{T}<0
$$

The initial distance to go $\rho_{0}$, the initial commanded velocity $\dot{\rho}_{0}<0$, and the final commanded arrival velocity $\dot{\rho}_{T}<0$ $\left(\left|\dot{\rho}_{0}\right|>\left|\dot{\rho}_{T}\right|\right)$, are all known. The slope $a$ is equal to

$$
a=\left(\dot{\rho}_{0}-\dot{\rho}_{T}\right) / \rho_{0}<0
$$

The commanded path, Eq. (26), corresponds to a varying commanded acceleration $\ddot{\rho}=a \dot{\rho}$, and since $|\dot{\rho}|$ decreases with time, the acceleration (i.e., actually deceleration) also decreases with time. These features of the glideslope scheme are desirable. With the boundary conditions, Eqs. (27) and
(28), the solution to Eq. (26) is

$$
\rho(t)=\rho_{0} e^{a t}+\left(\dot{\rho}_{T} / a\right)\left(e^{a t}-1\right)
$$

and the transfer time $T$ is

$$
T=(1 / a) \ln \left(\dot{\rho}_{T} / \dot{\rho}_{0}\right)
$$

where, $a<0$ and $\dot{\rho}_{T}<\dot{\rho}_{0}<0$. The scheme to move the chaser from $\boldsymbol{r}_{0}$ to $\boldsymbol{r}_{T}$ can be obtained as follows.

Let the number of thruster firings in time $T$ be $N$ and the uniform interval between any two pulses be $\Delta t=T / N$. The thrusters are thus fired at time $t_{m}=m \Delta t$ ( $m=0,1, \ldots, N-1)$, and the $m$ th pulse pushes the chaser from $\boldsymbol{r}_{m}\left(\rho=\rho_{m}\right)$ to $\boldsymbol{r}_{m+1}\left(\rho=\rho_{m+1}\right)$, where,

$$
\begin{aligned}
& \boldsymbol{r}_{m}=\boldsymbol{r}_{T}+\rho_{m} \boldsymbol{u}_{\rho} \\
& \rho_{m}=\rho\left(t_{m}\right)=\rho_{0} e^{a t_{m}}+\left(\dot{\rho}_{T} / a\right)\left(e^{a t_{m}}-1\right)
\end{aligned}
$$

The arrival velocity at $m$ th location is $\dot{r}_{m}^{-}$, and the departure velocity $\dot{\boldsymbol{r}}_{m}^{+}$to travel from $\boldsymbol{r}_{m}$ to $\boldsymbol{r}_{m+1}$ is

$$
\dot{\boldsymbol{r}}_{m}^{+}=\boldsymbol{\Phi}_{r \dot{r}}^{-1}\left(\boldsymbol{r}_{m+1}-\boldsymbol{\Phi}_{r r} \boldsymbol{r}_{m}\right)
$$

where, $\boldsymbol{\Phi}_{r r}=\boldsymbol{\Phi}_{r r}(\Delta t)$ and $\boldsymbol{\Phi}_{r \dot{r}}=\boldsymbol{\Phi}_{r \dot{r}}(\Delta t)$. The incremental velocity at $\boldsymbol{r}_{m}$ is then

$$
\Delta \boldsymbol{v}_{m}=\boldsymbol{\Phi}_{r \dot{r}}^{-1}\left(\boldsymbol{r}_{m+1}-\boldsymbol{\Phi}_{r r} \boldsymbol{r}_{m}\right)-\dot{\boldsymbol{r}}_{m}^{-}
$$

and the chaser will arrive at $\boldsymbol{r}_{m+1}$ with velocity $\dot{\boldsymbol{r}}_{m+1}^{-}$equal to

$$
\dot{\boldsymbol{r}}_{m+1}^{-}=\boldsymbol{\Phi}_{\dot{r r}}(\Delta t) \boldsymbol{r}_{m}+\boldsymbol{\Phi}_{\dot{r} r}(\Delta t) \dot{\boldsymbol{r}}_{m}^{+}
$$

The actual path of the chaser will not be along the vector $\rho$, of course, but rather will result from the differential spherical gravitational force in Clohessy-Wiltshire equations, the path illustrated in Fig. 3 with humps. Specifically, following Clohessy-Wiltshire equations the path is given by

$$
\boldsymbol{r}(t)=\boldsymbol{\Phi}_{r r}\left(t-t_{m}\right) \boldsymbol{r}_{m}+\boldsymbol{\Phi}_{r \dot{r}}\left(t-t_{m}\right) \dot{\boldsymbol{r}}_{m}^{+}
$$

Because the interval between any two successive pulses is the same, the spacecraft will move progressively slower as it approaches the target.

Because of maneuver correction, the revised augmented state vector and the corresponding covariance are as follows

$$
\begin{aligned}
& \boldsymbol{X}_{k}^{+}=\left(\boldsymbol{D}_{\Delta \hat{\boldsymbol{v}}_{k}} \Delta \hat{\boldsymbol{V}}_{\overline{\boldsymbol{x}}_{k}^{-c}}+\boldsymbol{D}_{\overline{\boldsymbol{x}}_{k}^{-c}}+\boldsymbol{I}\right) \boldsymbol{X}_{k}^{-} \\
& \boldsymbol{\Omega}_{k}^{+c}=\boldsymbol{M}_{k} \boldsymbol{\Omega}_{k}^{-c} \boldsymbol{M}_{k}^{\mathrm{T}}+\boldsymbol{N}_{k} \boldsymbol{P}_{k}^{\delta \boldsymbol{v}} \boldsymbol{N}_{k}^{\mathrm{T}}
\end{aligned}
$$

where $\boldsymbol{P}_{k}^{\delta v}$ is the maneuver execution error covariance matrix, the specific expressions of $\boldsymbol{M}_{k}$ and $\boldsymbol{N}_{k}$ are as follows

$\boldsymbol{M}_{k}=\left[\begin{array}{c:c}\boldsymbol{D}_{\overline{\boldsymbol{x}}_{k}^{-c}}+\boldsymbol{I}_{6 \times 6} & \boldsymbol{D}_{\Delta \hat{\boldsymbol{v}}_{k}} \Delta \hat{\boldsymbol{V}}_{\overline{\boldsymbol{x}}_{k}^{-c}} \\ \hdashline \mathbf{0}_{6 \times 6} & \boldsymbol{D}_{\Delta \hat{\boldsymbol{v}}_{k}} \Delta \hat{\boldsymbol{V}}_{\overline{\boldsymbol{x}}_{k}^{-c}}+\boldsymbol{D}_{\overline{\boldsymbol{x}}_{k}^{-c}}+\boldsymbol{I}_{6 \times 6}\end{array}\right]$

$\boldsymbol{N}_{k}=\left[\begin{array}{l}\boldsymbol{I}_{6 \times 6} \\ \mathbf{0}_{6 \times 6}\end{array}\right]$

where

$$
\boldsymbol{D}_{\overline{\boldsymbol{x}}_{k}^{-c}}=\left.\frac{\partial \boldsymbol{d}}{\partial \boldsymbol{X}}\right|_{\overline{\boldsymbol{x}}_{k}^{-c}} \text { and } \boldsymbol{D}_{\Delta \hat{\boldsymbol{v}}_{k}}=\left.\frac{\partial \boldsymbol{d}}{\partial \boldsymbol{v}}\right|_{\Delta \hat{\boldsymbol{v}}_{k}}
$$

are the differential of augmented state change, $\boldsymbol{d}\left(\overline{\boldsymbol{x}}_{k}^{-c}, \Delta \hat{\boldsymbol{v}}_{k}\right)$, respect to current augmented state and impulsive maneuver, respectively. As the impulsive assumption is used, i.e. the 
position will not change during maneuver, then $\boldsymbol{D}_{\overline{\boldsymbol{x}}_{k}^{-c}}$ equals

$$
\boldsymbol{D}_{\overline{\boldsymbol{x}}_{k}^{-c}}=\left.\frac{\partial \boldsymbol{d}}{\partial \boldsymbol{X}}\right|_{\overline{\boldsymbol{x}}_{k}^{-c}}=\left[\mathbf{0}_{6 \times 6}\right]
$$

and $\boldsymbol{D}_{\Delta \hat{v}_{k}}$ is

$$
\boldsymbol{D}_{\Delta \hat{v}_{k}}=\left.\frac{\partial \boldsymbol{d}}{\partial \boldsymbol{v}}\right|_{\Delta \hat{v}_{k}}=\left[\begin{array}{c}
\mathbf{0}_{3 \times 3} \\
\boldsymbol{I}_{3 \times 3}
\end{array}\right]
$$

$\Delta \hat{\boldsymbol{V}}_{\overline{\boldsymbol{x}}_{k}^{-c}}$ is derived by differentiating Eq. (35) respect to $\overline{\boldsymbol{x}}_{k}^{-c}=\left[\begin{array}{ll}\boldsymbol{r}_{k} & \dot{\boldsymbol{r}}_{k}^{-c}\end{array}\right]$,

$$
\Delta \hat{\boldsymbol{V}}_{\overline{\boldsymbol{x}}_{k}^{-c}}=\left.\frac{\partial \hat{\boldsymbol{v}}}{\partial \boldsymbol{X}}\right|_{\overline{\boldsymbol{x}}_{k}^{-c}}=\left[\begin{array}{ll}
-\boldsymbol{\Phi}_{r \dot{r}}^{-1} \boldsymbol{\Phi}_{r r} & -\boldsymbol{I}_{3 \times 3}
\end{array}\right]
$$

Finally, we achieve the complete analytical closed-loop linear covariance analysis model, which is feasible for onboard use to analysis the trajectory dispersion, evaluate the navigation performance as well as on-line maneuver planning.

\section{Numerical Simulation}

To validate the methods, the SwissCube TLE data from March 23, 2016 at UTC 13:45:16 was chosen to serve as the initial inertia state of the target: $a_{0}=7086121.3370 \mathrm{~m}$, $e_{0}=0.0007, \quad i_{0}=1.7176 \mathrm{rad}, \quad \Omega_{0}=5.0423 \mathrm{rad}, \quad \omega_{0}=$ $5.9745 \mathrm{rad}, f_{0}=0.31075 \mathrm{rad}$, and $\tau=6 \mathrm{~min}$. The initial relative position estimated error covariance matrix is $\hat{\boldsymbol{P}}_{r}=$ $\operatorname{diag}\left[\left(0.03 \rho_{0}\right)^{2},\left(0.015 \rho_{0}\right)^{2},\left(0.015 \rho_{0}\right)^{2}\right]^{\mathrm{T}} \mathrm{m}^{2}$, the initial relative velocity estimated error covariance matrix is $\hat{\boldsymbol{P}}_{v}=$ $10^{-4} \cdot \hat{\boldsymbol{P}}_{r} \mathrm{~m}^{2} / \mathrm{s}^{2}$, initial relative state dispersion covariance matrix is $\boldsymbol{P}=\left[\begin{array}{ll}\hat{\boldsymbol{P}}_{r} & \hat{\boldsymbol{P}}_{v}\end{array}\right]^{\mathrm{T}}$, the camera measurement noise covariance matrix is $\boldsymbol{R}=\operatorname{diag}\left[\left(3 \times 10^{-4} \mathrm{rad}\right)^{2}, \quad(3 \times\right.$ $\left.\left.10^{-4} \mathrm{rad}\right)^{2}\right]$, and the two different Lidar measurement noise covariance matrices are $\boldsymbol{R}_{L 1}=\operatorname{diag}\left[(3 \times 0.1 \mathrm{~m})^{2}, \quad(3 \times\right.$ $\left.\left.10^{-4} \mathrm{rad}\right)^{2},\left(3 \times 10^{-4} \mathrm{rad}\right)^{2}\right]$ and $\boldsymbol{R}_{L 2}=\operatorname{diag}\left[(0.1 \mathrm{~m})^{2},(3 \times\right.$ $\left.\left.10^{-4} \mathrm{rad}\right)^{2},\left(3 \times 10^{-4} \mathrm{rad}\right)^{2}\right]$. For the system noise covariance matrix $\boldsymbol{Q}$, refer to Eq. (41) of Hari et al., ${ }^{38)}$ with a propagation step size of $1 \mathrm{~s}$, a measurement frequency of $1 \mathrm{~Hz}$, and assuming that the target can be measured by the camera during the entire process.

\subsection{Simulation Case 1}

To validate and compare the effectiveness of a close-range AON observation model, a $0.5 \mathrm{~m}$ normal offset (i.e., $\left.\boldsymbol{r}_{c m}=[0,0,0.5 \mathrm{~m}]^{\mathrm{T}}\right)$ is used for $-100 \mathrm{~m}$ relative stationkeeping observation. Figure 4 shows 100 Monte Carlo simulations using a traditional observation model and offset observation model for AON, respectively. After filtering approximately 0.6 periods, the $3 \sigma$ range estimation error decreased two orders (i.e., from initial decameter-level to final decimeter-level). However, using the traditional observation model, the $3 \sigma$ range estimation error remains constant, at the decameter-level. This proves that the offset observation model proposed solves the range-observability problem effectively.

\subsection{Simulation Case 2}

To validate the feasibility of AON, a 10-impulsive $-500 \mathrm{~m}$ to $-2 \mathrm{~m} v$-bar quasi-linear glide approach scenario is built.

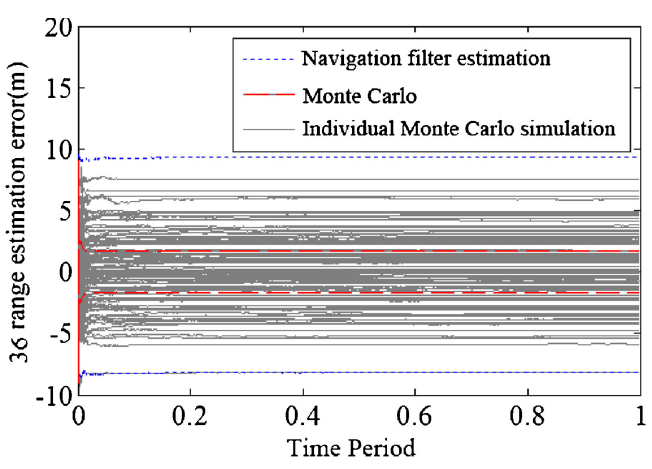

(a) Traditional observation model

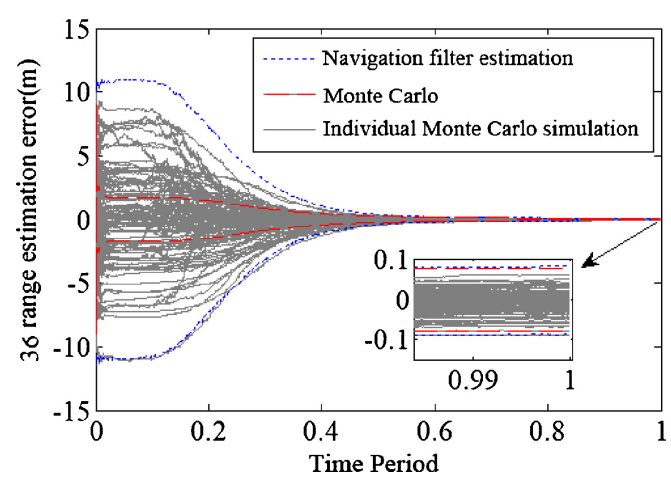

(b) Offset observation model

Fig. 4. Observation models comparison.

Two different range measurement accuracy Lidar-based navigation systems are selected for comparison. The initial nominal relative state is $\overline{\boldsymbol{x}}_{0}=[-500 \mathrm{~m}, 0 \mathrm{~m}, 0 \mathrm{~m}, 1 \mathrm{~m} / \mathrm{s}, 0 \mathrm{~m} / \mathrm{s}$, $0 \mathrm{~m} / \mathrm{s}]^{\mathrm{T}}$. The initial estimated relative state is $\hat{\boldsymbol{x}}_{0}=$ $[-483.751 \mathrm{~m}, 5.23 \mathrm{~m},-7.851 \mathrm{~m}, 0.874 \mathrm{~m} / \mathrm{s}, 0.018 \mathrm{~m} / \mathrm{s}$, $0.035 \mathrm{~m} / \mathrm{s}]^{\mathrm{T}}$. The final desired relative state is $\overline{\boldsymbol{x}}_{F}=$ $[-2 \mathrm{~m}, 0 \mathrm{~m}, 0 \mathrm{~m}, 0.01 \mathrm{~m} / \mathrm{s}, 0 \mathrm{~m} / \mathrm{s}, 0 \mathrm{~m} / \mathrm{s}]^{\mathrm{T}}$. The total flight time is $2316.540 \mathrm{~s}$, with a maneuver time array of $\boldsymbol{t}=$ $[0 \mathrm{~s}, 257.39 \mathrm{~s}, 514.79 \mathrm{~s}, 772.18 \mathrm{~s}, 1029.57 \mathrm{~s}, 1286.97 \mathrm{~s}$, $1544.36 \mathrm{~s}, 1801.75 \mathrm{~s}, 2059.15 \mathrm{~s}, 2316.54 \mathrm{~s}]$. The nominal relative state evolution over time is shown in Fig. 5. The relative state navigation deviation and control deviation evolution are shown in Figs. 6 and 7, respectively. The final relative state navigation error and control error statistical result of 100 Monte Carlo simulations are shown in Tables 1 and 2 , respectively.

Here, $\sigma_{\rho}$ is defined as the standard deviation of range. In Table 1, the statistical final relative state navigation errors using AON, Lidar $1\left(3 \sigma_{\rho}=0.3 \mathrm{~m}\right)$ and Lidar $2\left(3 \sigma_{\rho}=\right.$ $0.1 \mathrm{~m}$ ) are achieved based on 100 Monte Carlo simulations. It shows the final relative state navigation error difference between AON and Lidar is mainly in the local horizontal component of position: $7.6 \%$ (Lidar 1 is used) smaller than a Lidar with $0.3 \mathrm{~m}\left(3 \sigma_{\rho}\right)$ range measurement accuracy and almost twice that of a Lidar with $0.1 \mathrm{~m}\left(3 \sigma_{\rho}\right)$ range measurement accuracy, the navigation error difference on other relative state components is negligible. In fact, this result reveals that, in a multi-pulse glideslope guidance-based v-bar quasi-linear approach, $\mathrm{AON}$ can achieve the equivalent navigation accuracy of a Lidar having $0.3 \mathrm{~m}\left(3 \sigma_{\rho}\right)$ range measurement accuracy 
Trans. Japan Soc. Aero. Space Sci., Vol. 63, No. 2, 2020
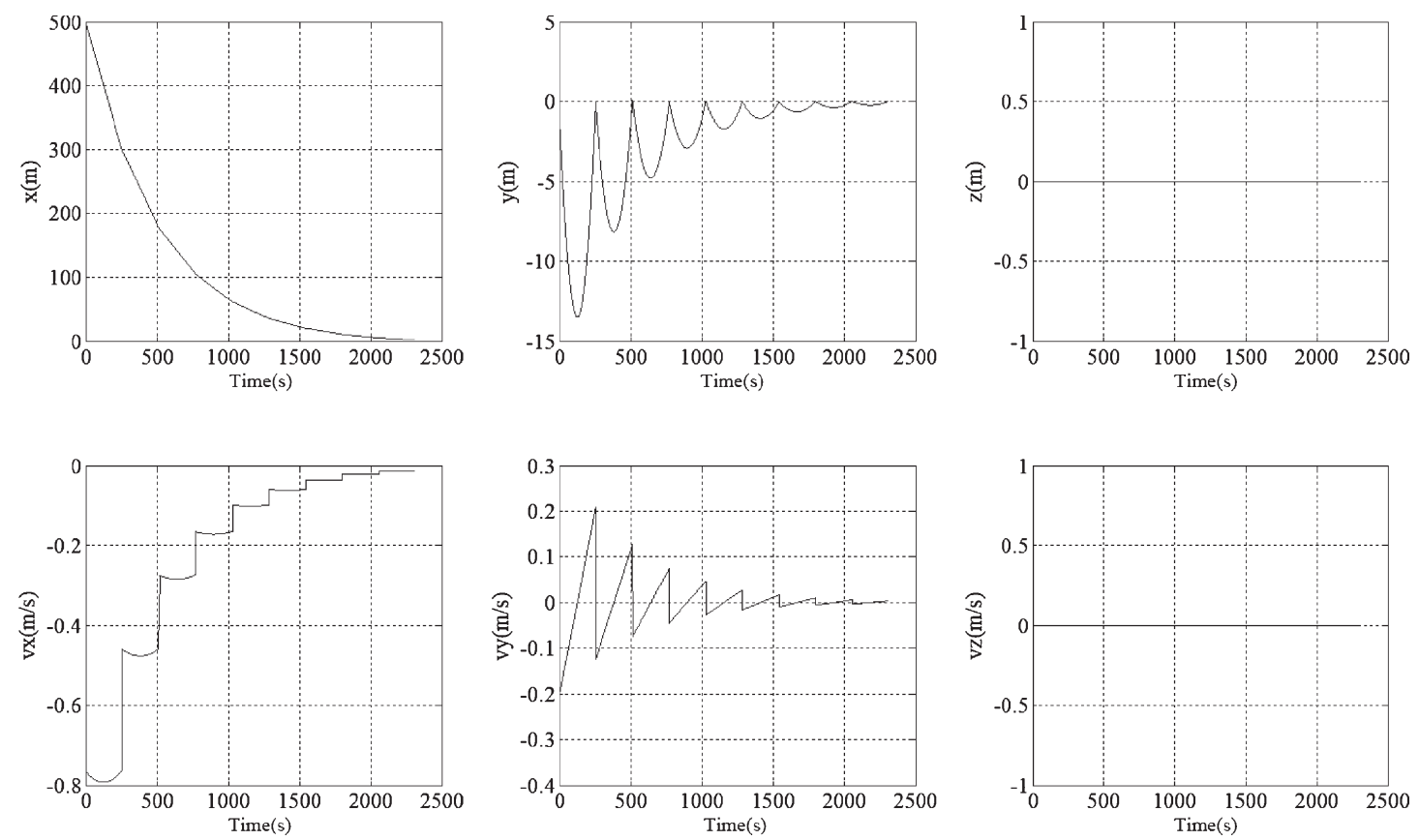

Fig. 5. Nominal relative state evolution.
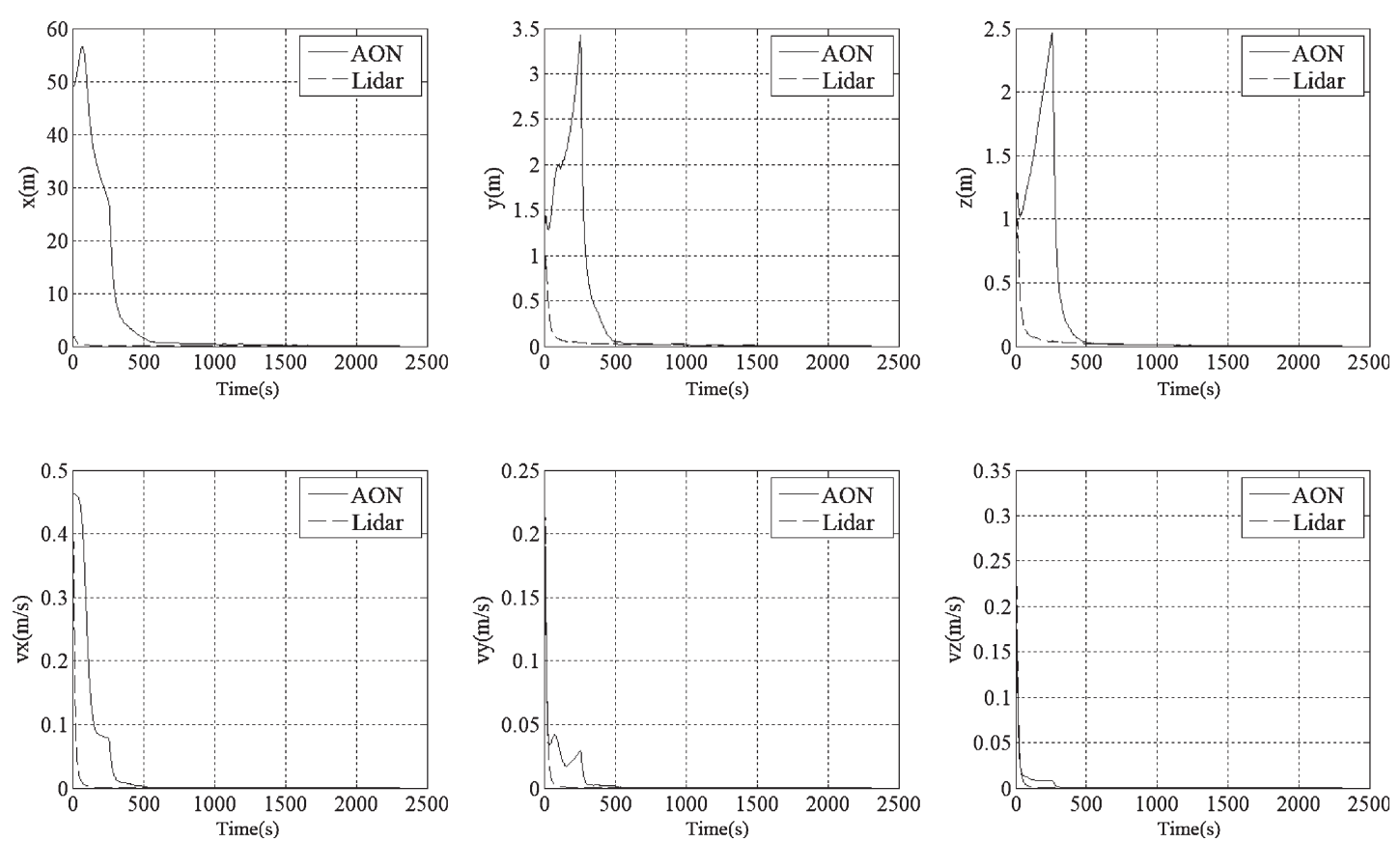

Fig. 6. Relative state navigation deviation evolution.

Table 1. Statistical final navigation error of Monte Carlo simulations.

\begin{tabular}{lcccccc}
\hline Strategy & $\begin{array}{c}3 \sigma_{x} \\
(\mathrm{~m})\end{array}$ & $\begin{array}{c}3 \sigma_{y} \\
(\mathrm{~m})\end{array}$ & $\begin{array}{c}3 \sigma_{z} \\
(\mathrm{~m})\end{array}$ & $\begin{array}{c}3 \sigma_{\dot{x}} \\
(\mathrm{~m} / \mathrm{s})\end{array}$ & $\begin{array}{c}3 \sigma_{\dot{y}} \\
(\mathrm{~m} / \mathrm{s})\end{array}$ & $\begin{array}{c}3 \sigma_{\dot{z}} \\
(\mathrm{~m} / \mathrm{s})\end{array}$ \\
\hline AON & 0.0754 & 0.0010 & 0.0013 & $2.37 \mathrm{e}-4$ & $9.69 \mathrm{e}-5$ & $8.93 \mathrm{e}-5$ \\
Lidar 1 & 0.0816 & $8.81 \mathrm{e}-4$ & 0.0010 & $3.31 \mathrm{e}-4$ & $8.64 \mathrm{e}-5$ & $1.45 \mathrm{e}-4$ \\
Lidar 2 & 0.0379 & $8.69 \mathrm{e}-4$ & 0.0009 & $3.41 \mathrm{e}-4$ & $1.02 \mathrm{e}-4$ & $1.03 \mathrm{e}-4$ \\
\hline
\end{tabular}

and same angular measure accuracy. This means that using a well-designed relative approach guidance profile, the camera operational range can be extended, which would enable the operating time of the Lidar to be shortened, or even replaced, by an optimal/infrared camera as the relative navigation sensor for close-in proximity operations. This would be very helpful in saving chaser mass and power.

Figure 6 shows the relative state navigation deviation evolution comparison of Lidar-based navigation and AON, which are noted by dashed and solid lines, respectively. Lidar shows rapid convergence after $180 \mathrm{~s}$ of observation. However, AON takes about $600 \mathrm{~s}$, with three maneuvers applied to converge to a stable error level, and further gradually achieving equivalent position estimation accuracy as the Lidar after 1,500 s, with six maneuvers applied.

Figure 7 shows the relative state control deviation evolu- 

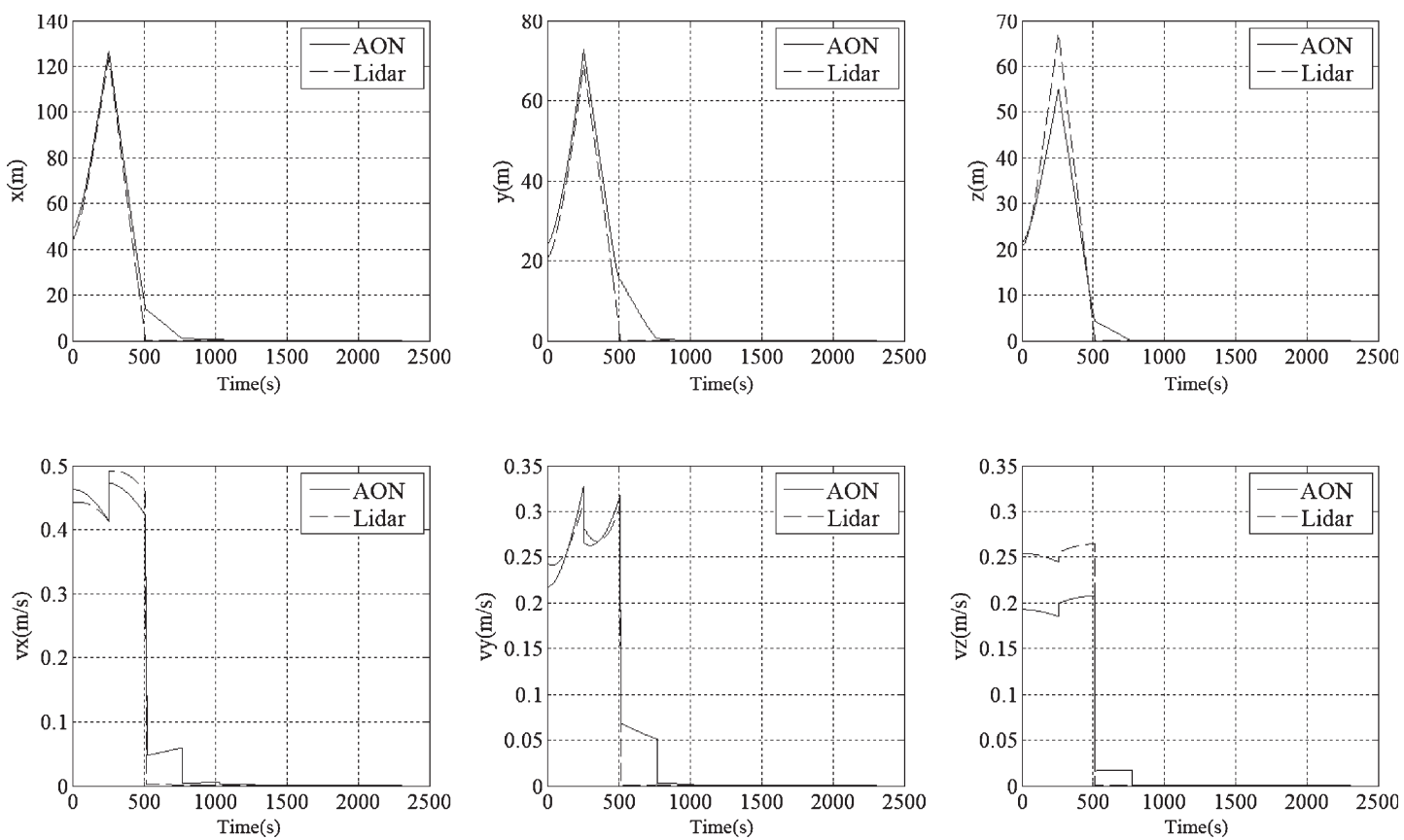

Fig. 7. Relative state control deviation evolution. (Lidar 1 is used.)

Table 2. Statistical final control error of Monte Carlo simulations.

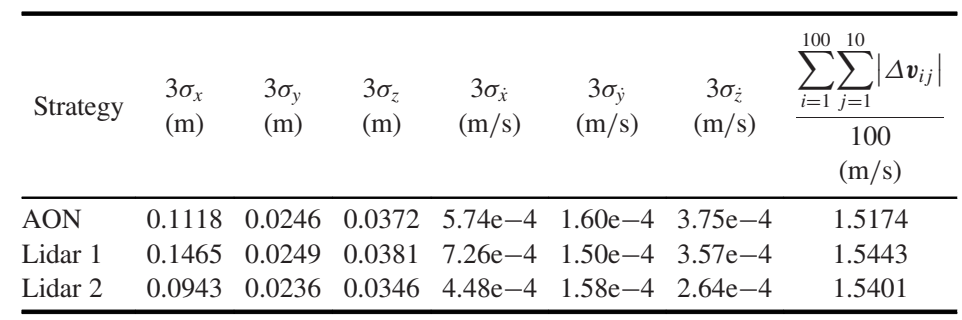

tion comparison for Lidar-based navigation and AON. After three maneuver corrections, the relative state control error is reduced to stable accuracy, which is specifically shown in the third row of Table 2. However, when AON is utilized, two more maneuver corrections are needed to improve the control accuracy when compared to the Lidar-based situation, as shown in the second row of Table 2. Furthermore, from Table 2, the final relative state control error difference between AON and Lidar is mainly in the local horizontal component of position (i.e., $23.69 \%$ smaller than a Lidar with $0.3 \mathrm{~m}\left(3 \sigma_{\rho}\right)$ range measurement accuracy and $18.56 \%$ larger than a Lidar with $0.1 \mathrm{~m}\left(3 \sigma_{\rho}\right)$ range measurement accuracy. The control error difference on other relative state components is negligible. Additionally, the rate of fuel consumption is very close. This reveals that using maneuver correction can improve navigation accuracy and further reduce the control error differences between Lidar-based navigation and AON, and will not increase fuel consumption.

\subsection{Simulation Case 3}

To evaluate the effectiveness of filter tuning, the previous scenario was repeated using a tuned SRUKF filter. In Fig. 8, the tuned and standard SRUKF AON-based 100 Monte Carlo simulations and closed-loop linear covariance analysis are compared. In the graphs, the red line shows the relative tra- jectory in the $x z$ projected plane and the gray lines show the 100 simulated trajectories, respectively. The black ellipses give the closed-loop control error ellipse. The blue dots are the sample relative positions for each error ellipse. All of the sample trajectories locate in the ellipse, which verifies the accuracy and validity of the closed-loop linear covariance analysis method. Furthermore, using tuned SRUKF, we find that trajectory dispersion already converges to the sub-meter level at $100 \mathrm{~m}$ from the target; this is two orders improved compared with the standard SRUKF situation. Additionally, the final sample points using the tuned SRUKF are better matched with the covariance estimation. However, covariance estimation using the standard SRUKF is a bit pessimistic; that is, the dispersions estimated are larger than the real dispersions. Accordingly, by tuning the filter, a more robust trajectory and accurate covariance prediction are achieved. This is important and useful for close-in proximity operation, onboard maneuver planning and real-time closedloop control error estimation.

\subsection{Simulation Case 4}

To compare the influence of nominal trajectory on control accuracy, trajectory robustness and fuel consumption, an approach-circumnavigate-approach trajectory is utilized for comparison. The proximity operation relative trajectory con- 


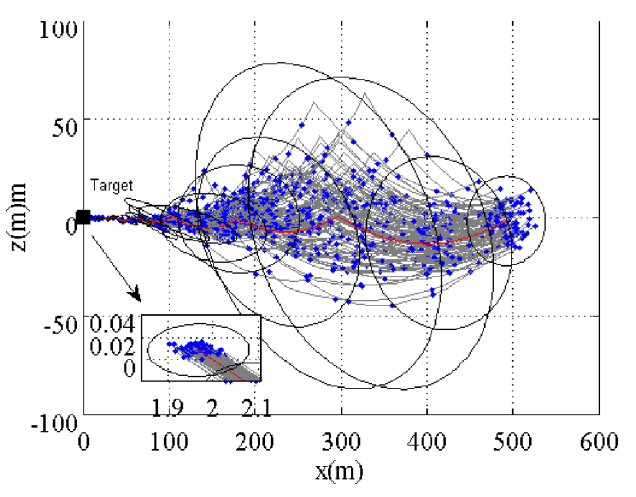

(a) Standard SRUKF based AON

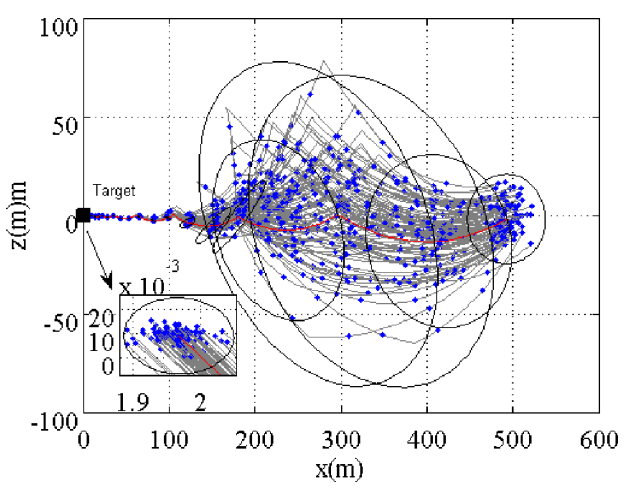

(b) Tuned SRUKF-based AON

Fig. 8. Monte Carlo simulation and closed-loop linear covariance analysis.

sists of three parts. Firstly, a 10-impulse v-bar quasi-linear glide approach from $500 \mathrm{~m}$ to $100 \mathrm{~m}$, followed by a 20 impulse circumnavigate (i.e., the fly-around radius is $100 \mathrm{~m}$ ), and this is further followed by a 10-impulse v-bar quasi-linear glide approach, from $100 \mathrm{~m}$ to $20 \mathrm{~m}$. Using the tuned SRUKF AON, the 100 Monte Carlo simulations and closed-form linear covariance analyses are shown in Fig. 9.

This shows that, after the first glide approach, the trajectory dispersion already converges to the sub-meter level. During circumnavigation, the dispersion keeps converging slightly, and finally achieves $3 \sigma$ relative position dispersion better than $0.1 \mathrm{~m}$ after the second glide approach. Circumnavigation is always used for uncooperative target investigation, and transfer to the final approach direction for catching. This proves that AON-based glide approach-circumnavigate-glide approach is feasible and safe for close-in proximity operation.

\section{Conclusion}

In this paper, the feasibility of angles-only navigationbased close-in proximity operation is studied for upcoming small satellite-based uncooperative rendezvous missions, such as active debris removal, on-orbit servicing and inspection.

The SRUKF method is adapted for relative state estimations from the perspective of low-volume/mass, power savings, and low-cost optical/infrared camera observations, along with Lidar measurements. Considering the simplicity and achievements in engineering, a multi-pulse glideslope guidance law is utilized. Using a linear covariance technique,

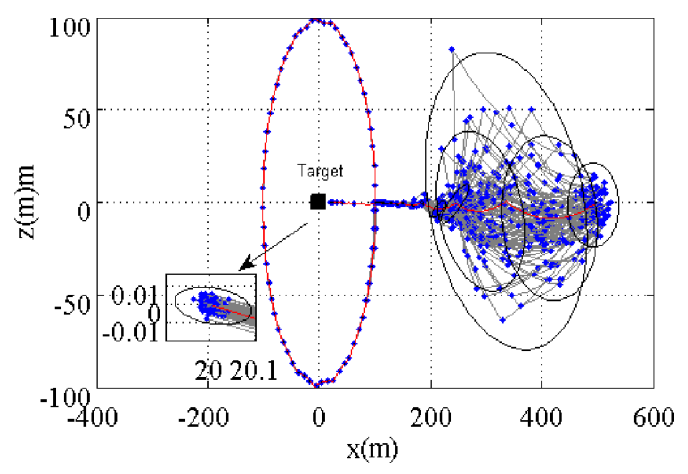

Fig. 9. Glide approach-circumnavigate-glide approach.

a complete set of analytical functions are deduced for closedloop true dispersions and estimated dispersion analysis. Through Monte Carlo simulation, it has been proven that using the offset observation model proposed for close-in proximity operation, after about 0.6 periods of filtering, the $3 \sigma$ range estimation error decreases from the initial decameterlevel to a final decimeter-level, providing a good solution for the range-observability problem.

Considering both AON observability and fuel consumption, two proximity operation mission trajectories are designed: a direct v-bar quasi-linear glide approach for robotic arm capture or net capture, and a glide approach-circumnavigateglide approach for capture with an attitude adjustment requirement. A chaser equipped with a normal quality $\left(\sigma_{\theta}=\right.$ $3 \times 10^{-4} \mathrm{rad}$ ) camera using a 10 -impulsive v-bar quasilinear glide approach from $500 \mathrm{~m}$ to $2 \mathrm{~m}$ can achieve a comparative relative state estimation and control accuracy equivalent to a Lidar with $0.3 \mathrm{~m}\left(3 \sigma_{\rho}\right)$ range measurement accuracy after filtering for approximately $1.000 \mathrm{~s}$ and five maneuver corrections, which is about twice that of the Lidar. This reveals that using a well-designed relative approach guidance profile, the camera operational range can be extended, and the Lidar operation time can be shorten or even replaced by an optimal/infrared camera, which would be very helpful in saving mass and power for the chaser.

Monte Carlo simulation verified the validity of the closedloop linear covariance analysis method and revealed that the variable-structure SRUKF proposed leads to a more robust trajectory: The true dispersion is improved two orders in the $100 \mathrm{~m}$ range, there is more accurate covariance prediction, and all of the sample trajectories locate in the ellipse as compared with the standard SRUKF. The analytical closed-loop linear covariance analysis method proposed is important and useful for close-in proximity operation, onboard maneuver planning and real-time closed-loop control error estimation.

A more efficient optimization algorithm and SRUKF sigma point sampling strategy will be explored to develop onboard guidance, navigation and control algorithms for small-satellite autonomous rendezvous missions. 


\section{Acknowledgments}

Research supported by the National Natural Science Foundation of China, No. 91438202 , No. 61690212 and No. 61833009.

The authors declare that there is no conflict of interest regarding the publication of this paper.

All data included in this study are available upon request by contacting the corresponding author.

\section{References}

1) Wormnes, K., Le Letty, R., Summerer, L., Schonenborg, R., DuboisMatra, O., Luraschi, E., Cropp, A., Krag, H., and Delaval, J.: ESA Technologies for Space Debris Remediation, Proceedings of the 6th IAASS Conference: Safety Is Not an Option, Apr. 2013, pp. 3-4.

2) Esmiller, B., Jacquelard, C., Eckel, H.-A., and Wnuk, E.: Space Debris Removal by Ground-based Lasers: Main Conclusions of the European Project CLEANSPACE, Appl. Optics, 53, 31 (2014), pp. 145-154.

3) Pelton, J. N.: Current Space Debris Remediation and On-orbit Servicing Initiatives, New Solutions for the Space Debris Problem, Springer International Publishing, 2015, pp. 11-29.

4) Chari, R. J.: Autonomous Orbital Rendezvous Using Angles-Only Navigation, Master Dissertation, Department of Aeronautics and Astronautics, Massachusetts Institute of Technology, 2001.

5) Geller, D. K.: Analysis of the Relative Attitude Estimation and Control Problem for Satellite Inspection and Orbital Rendezvous, J. Astronaut. Sci., 55, 2 (2007), pp. 195-214.

6) Woffinden, D. C. and Geller, D. K.: Optimal Orbital Rendezvous Maneuvering for Angles-only Navigation, J. Guid. Control Dynam., 32, 4 (2009), pp. 1382-1387.

7) Woffinden, D. C. and Geller, D. K.: Observability Criteria for Anglesonly Navigation, IEEE Trans. Aerospace and Electronic Systems, 45, 3 (2009), pp. 1194-1208.

8) Woffinden, D. C.: Angles-only Navigation for Autonomous Orbital Rendezvous, ProQuest, 2008.

9) Kannan, S. K., Johnson, E. N., Watanabe, Y., and Sattigeri, R.: VisionBased Tracking of Uncooperative Targets, Int. J. Aerospace Eng., 2011 (2011), Article ID 243268, 17 pages. doi:10.1155/2011/243268

10) Klein, I. and Geller, D. K.: Zero Delta-V Solution to the Angles-Only Range Observability Problem during Orbital Proximity Operations, Advances in Estimation, Navigation, and Spacecraft Control, Choukroun, D., Oshman, Y., Thienel, J., and Idan, M. (eds.), Springer, Berlin, Heidelberg, 2015, pp. 351-369.

11) LeGrand, K. A., Kyle, J. D., and Henry, J. P.: Bearings-Only Initial Relative Orbit Determination, J. Guid. Control Dynam., 38, 9 (2015), pp. 1699-1713.

12) Li, J. R., Li, H. Y., Tang, G. J., and Luo, Y. Z.: Research on the Strategy of Angles-only Relative Navigation for Autonomous Rendezvous, Sci. China Technol. Sci., 54, 7 (2011), pp. 1865-1872.

13) Tombasco, J. and Penina, A.: Along-Track Separation Uncertainty Modeling Given Space-Based Optical Observations, J. Guid. Control Dynam., 35, 3 (2012), pp. 732-739.

14) Grzymisch, J. and Walter, F.: Analytic Optimal Observability Maneuvers for In-Orbit Bearings-Only Rendezvous, J. Guid. Control Dynam., 37, 5 (2014), pp. 1658-1664.

15) Noteborn, R., Bodin, P., Larsson, R., and Chasset, C.: Flight Results from the PRISMA Optical Line of Sight Based Autonomous Rendezvous Experiment, 4th International Conference on Spacecraft Formation Flying Missions \& Technologies, St-Hubert, Canada, 2011.

16) Bodin, P., Noteborn, R., Larsson, R., Karlsson, T., D’Amico, S., Ardaens, J.-S., Delpech, M., and Berges, J.-C.: The Prisma Formation Flying Demonstrator: Overview and Conclusions from the Nominal Mission, Adv. Astronaut. Sci., 144 (2012), pp. 441-460.

17) Delpech, M., Berges, J.-C., Karlsson, T., and Malbet, F.: Results of PRISMA/FFIORD Extended Mission and Applicability to Future Formation Flying and Active Debris Removal Missions, Int. J. Space Sci. Eng., 1, 4 (2013), pp. 382-409.

18) D'Amico, S.: Noncooperative Rendezvous Using Angles-only Optical
Navigation: System Design and Flight Results, J. Guid. Control Dynam., 36, 6 (2013), pp. 1576-1595.

19) Spurmann, J.: Spiraling Approach for Angles-Only Navigation within On-Orbit Servicing Missions, EuroGNC Conference, 2011, pp. 465-472.

20) Pi, J. and Bang, H.: Trajectory Design for Improving Observability of Angles-Only Relative Navigation between Two Satellites, J. Astronaut. Sci., 61, 4 (2014), pp. 391-412.

21) Huang, W.: Solving Coplanar Power-Limited Orbit Transfer Problem by Primer Vector Approximation Method, Int. J. Aerospace Eng., 2012 (2012), Article ID 480320, 9 pages. doi:10.1155/2012/480320

22) Simeoni, F., Casalino, L., Zavoli, A., and Colasurdo, G.: Indirect Optimization of Satellite Deployment into a Highly Elliptic Orbit, Int. J. Aerospace Eng., 2012 (2012), Article ID 152683, 14 pages. doi:10.1155/2012/152683

23) Tang, G. J., Luo, Y. Z., and Li, H. Y.: Optimal Robust Linearized Impulsive Rendezvous, Aerospace Sci. Technol., 11 (2007), pp. 563-569.

24) Luo, Y. Z., Yang, Z., and Li, H. N.: Robust Optimization of Nonlinear Impulsive Rendezvous with Uncertainty, Sci. China Phys. Mech. Astronomy, 54, 4 (2014), pp. 731-740.

25) Luo, Y. Z.: Research on Space Optimal Rendezvous Trajectory Planning Approach, Doctor Dissertation, College of Aerospace Science and Engineering, National University of Defense Technology, Changsha, China, 2007.

26) Luo, Y., Liang, L., Niu, Z., and Tang, G.: Safety-Optimal Linearized Impulsive Rendezvous with Trajectory Uncertainties, J. Aerospace Eng., 27, 6 (2014), pp. 391-412.

27) Li, J. R., Li, H. Y., and Tang, G. J.: Optimal Multi-objective Trajectory Design Based on Close-looped Control for Autonomous Rendezvous, Sci. China Technol. Sci., 54, 11 (2011), pp. 3091-3097.

28) Alfriend, K., Vadali, S. R., Gurfil, P., How, J., and Breger, L.: Spacecraft Formation Flying: Dynamics, Control and Navigation, Vol. 2, Butterworth-Heinemann, 2009, pp. 105-110.

29) Yamanaka, K. and Ankersen, F.: New State Transition Matrix for Relative motion on an Arbitrary Elliptical Orbit, J. Guid. Control Dynam., 25, 1 (2002), pp. 60-66.

30) Julier, S., Uhlmann, J., and Durrant-Whyte, H. F.: A New Approach for Filtering Nonlinear Systems, American Control Conference, Proceedings of the IEEE, Vol. 3, 1995, pp. 1628-1632.

31) Julier, S. J. and Uhlmann, J. K.: A New Extension of the Kalman Filter to Nonlinear Systems, Proc. of AeroSense: The 11th Int. Symp. on Aerospace/Defence Sensing, Simulation and Controls, 1997.

32) Julier, S. and Uhlmann, J.: A New Method for the Nonlinear Transformation of Means and Covariances in Filters and Estimator, IEEE Trans. AC, 45, 3 (2000), pp. 477-478.

33) van der Merwe, R. and Wan, E. A.: The Square-root Unscented Kalman Filter for State and Parameter Estimation, Proceedings of the International Conference on Acoustics, Speech, and Signal Processing, Salt Lake City, Utah, USA, Vol. 6, 2001, pp. 3461-3464.

34) Liu, B., Chen, Z., Liu, X., and Yang, F.: An Efficient Nonlinear Filter for Spacecraft Attitude Estimation, Int. J. Aerospace Eng., 2014 (2014), Article ID 540235, 11 pages. doi:10.1155/2014/540235

35) Zhou, Y., Zhang, C., Zhang, Y., and Zhang, J.: A New Adaptive Square-Root Unscented Kalman Filter for Nonlinear Systems with Additive Noise, Int. J. Aerospace Eng., 2015 (2015), Article ID 381478, 9 pages. doi:10.1155/2015/381478

36) de Mijolla, L., Cavrois, B., Profizi, A., Renault, C., and Cropp, A.: Covariance Analysis Tool for Far Non-Cooperative Rendezvous, AIAA Guidance, Navigation, and Control Conference, AIAA, Boston, MA, 2013, pp. 1-16.

37) Geller, D. K.: Linear Covariance Techniques for Orbital Rendezvous Analysis and Autonomous Onboard Mission Planning, J. Guid. Control Dynam., 29, 6 (2006), pp. 1404-1414.

38) Hari, B. H., Myron, L. T., and Dana-Bashian, D. J.: Guidance and Relative Navigation for Autonomous Rendezvous in a Circular Orbit, J. Guid. Control Dynam., 25, 3 (2002), pp. 553-562. 